Pathologe 2017 · 38:105-111 DOI 10.1007/s00292-017-0275-z

Online publiziert: 27. Februar 2017

(c) Der/die Autor(en) 2017. Dieser Artikel ist eine Open-Access-Publikation.

Schwerpunktherausgeber

G. Baretton, Dresden

CrossMark

Es ist bislang ungeklärt, von welcher Mindestgröße an eine Vermehrung von CD117-positiven interstitiellen Cajal-Zellen (ICC) in der Muscularis propria des Gastrointestinaltrakts als gastrointestinaler Stromatumor (GIST) bewertet werden sollte. Kleine GIST mit einem maximalen Durchmesser von $1 \mathrm{~cm}$ werden auch als mikroskopische GIST bezeichnet und sind wesentlich häufiger als klinisch relevante GIST. Ein Rückfallrisiko besteht bei den kleinen Tumoren im Allgemeinen nicht. Bereits mikroskopische GIST tragen als sehr frühes pathogenetisches Ereignis und als Indikator einer echten Neoplasie mehrheitlich Mutationen im KIT- oder PDGFRA-Gen. Multiple GIST können auf eine hereditäre oder syndromale Genese hinweisen oder Ausdruck einer Metastasierung sein, selten kann es sich aber auch um synchrone oder metachrone sporadische Tumoren handeln. Die adäquate Zuordnung hat erhebliche klinischtherapeutische Konsequenzen.

\section{„Kleiner GIST": Hyperplasie der interstitiellen Cajal- Zellen oder gastrointestinaler Stromatumor?}

Gastrointestinale Stromatumoren(GIST) sind die häufigsten mesenchymalen $\mathrm{Tu}$ moren des Verdauungstrakts, die mit ca. $60 \%$ am häufigsten im Magen auftreten, gefolgt vom Dünndarm mit etwa $30 \%$ und seltener im Rektum oder noch seltener im Ösophagus. Außerdem existieren intraabdominelle GIST ohne Beziehung zum tubulären Gastrointestinaltrakt, die auch als extragastro-

E. Wardelmann · W. Hartmann · M. Trautmann · J. Sperveslage · S. Elges ·

E. Hekeler · S. Huss

Gerhard-Domagk-Institut für Pathologie, Universitätsklinikum Münster, Münster, Deutschland

\title{
Gastrointestinale Stromatumoren des Magens und ihre Vorstufen
}

intestinale GIST (E-GIST) bezeichnet werden [1]. Viel häufiger nehmen diese Tumoren ihren Ausgang von der glattmuskulären Wandung des tubulären GI-Trakts und zwar mutmaßlich von den interstitiellen Cajal-Zellen (ICC) in Nachbarschaft des Plexus myentericus bzw. einer gemeinsamen weniger differenzierten Vorläuferzelle.

Die Inzidenz kleiner GIST mit einem Durchmesser von $1 \mathrm{~cm}$ oder weniger liegt bei systemischer Aufarbeitung von Autopsiemägen oder Gastrektomiepräparaten weit höher als die von klinisch relevanten größeren Tumoren. In vollständig aufgearbeiteten Gastrektomiepräparaten bzw. Autopsiemägen zweier Studien aus Japan und Deutschland [2, 3 ] konnten in $35 \%$ bzw. $22,5 \%$ aller Fälle sogenannte asymptomatische Mikro-GIST $\quad(0,2-10 \mathrm{~mm} \quad$ Durchmesser $)$ nachgewiesen werden. Demgegenüber ist die Zahl klinisch relevanter gastrointestinaler Stromatumoren mit ca. 15 bis 20 Neuerkrankungen/1 Mio. Einwohner und Jahr deutlich niedriger $[4,5]$. Gerade kleine GIST zeigen oft eine sehr enge räumliche Beziehung und Verzahnung mit der umgebenden Muscularis propria (•Abb. 1), möglicherweise als Ausdruck einer parakrinen Interaktion zwischen Muskelzellen und ICC in der Frühentwicklung von GIST. Größere Tumoren verlieren diese enge Beziehung zur glatten Muskulatur dann mit zunehmender Größe zugunsten eines zumeist expansiven verdrängenden Wachstums gegenüber der Umgebung.

Die Nomenklatur gerade der kleinen Läsionen ist in der Literatur variabel, weil unklar ist, bis wann noch von einer Hyperplasie der ICC im Sinne einer reinen und reversiblen Zellvermehrung und $\mathrm{ab}$ wann von einer echten Neoplasie ausgegangen werden kann. Eine Studie an 3 Patientinnen mit familiären, multiplen GIST erbrachte mithilfe der Untersuchung des HUMARA-Lokus auf Klonalität als Größengrenze zwischen ICC-Hyperplasie und GIST für Letzteren einen Durchmesser von $>5 \mathrm{~mm}$ [6]. Es wurden allerdings nur Fälle mit vermutlich hereditärer Disposition untersucht, während kleine sporadische Tumoren nicht zur Untersuchung kamen. Aktuell werden für die Frühformen von GIST Begriffe wie „sporadische ICC-Hyperplasie“ [7], „GIST tumorlet“ [3], „mikroskopischer GIST“ $[2,8]$ oder „minimaler GIST“ [9] verwendet. Der Begriff der ICC-Hyperplasie taucht heute eher im Kontext hereditärer Dispositionen z. B. im Rahmen der Neurofibromatose Typ I oder bei Keimbahnmutationen im KITGen auf. Bei diesen Patienten treten häufig neben manifesten GIST von relevanter Größe an zahlreichen weiteren Stellen des Gastrointestinaltrakts kleinste inzidentelle ICC-Knötchen als mutmaßliche Vorläufer auf [6]. Neben den nodulären ICC-Proliferationen können häufiger auch eher lineare ICC-Vermehrungen beobachtet werden, die zudem äußerst selten auch sporadisch beobachtet werden [10]. In unserem eigenen Register mit mehr als 3000 GIST konnten wir ein derartiges Phänomen bei 3 Patienten beobachten, exemplarisch ist ein linearer GIST des Ileums in • Abb. 2 dargestellt.

\section{KIT-/PDGFRA-/BRAF- \\ Mutationen in der Pathogenese von GIST}

Es ist durch zahlreiche Studien belegt, dass in $85-90 \%$ aller GIST als wesent- 

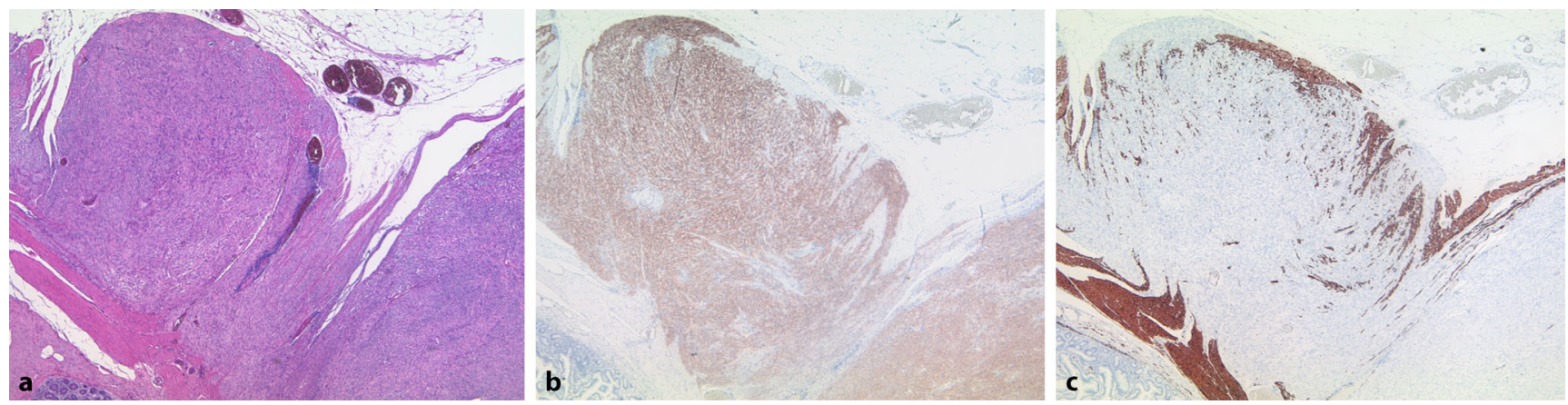

Abb. 1 Enge räumliche Beziehung kleiner gastrointestinaler Stromatumoren (GIST) mit der umgebenden Muscularis propria. (a, HE-Färbung, Vergrößerung 100:1) erkennt man einen kleinen spindelzelligen GIST, der an seiner Zirkumferenz eng mit der glatten Muskulatur interagiert. Dies kann möglicherweise ein Ausdruck einer parakrinen Interaktion zwischen Muskel- und Cajal-Zellen sein. Die Tumorzellen exprimieren KIT (b, Vergrößerung 100:1), die Muskulatur exprimiert Desmin (c, Vergrößerung 100:1)
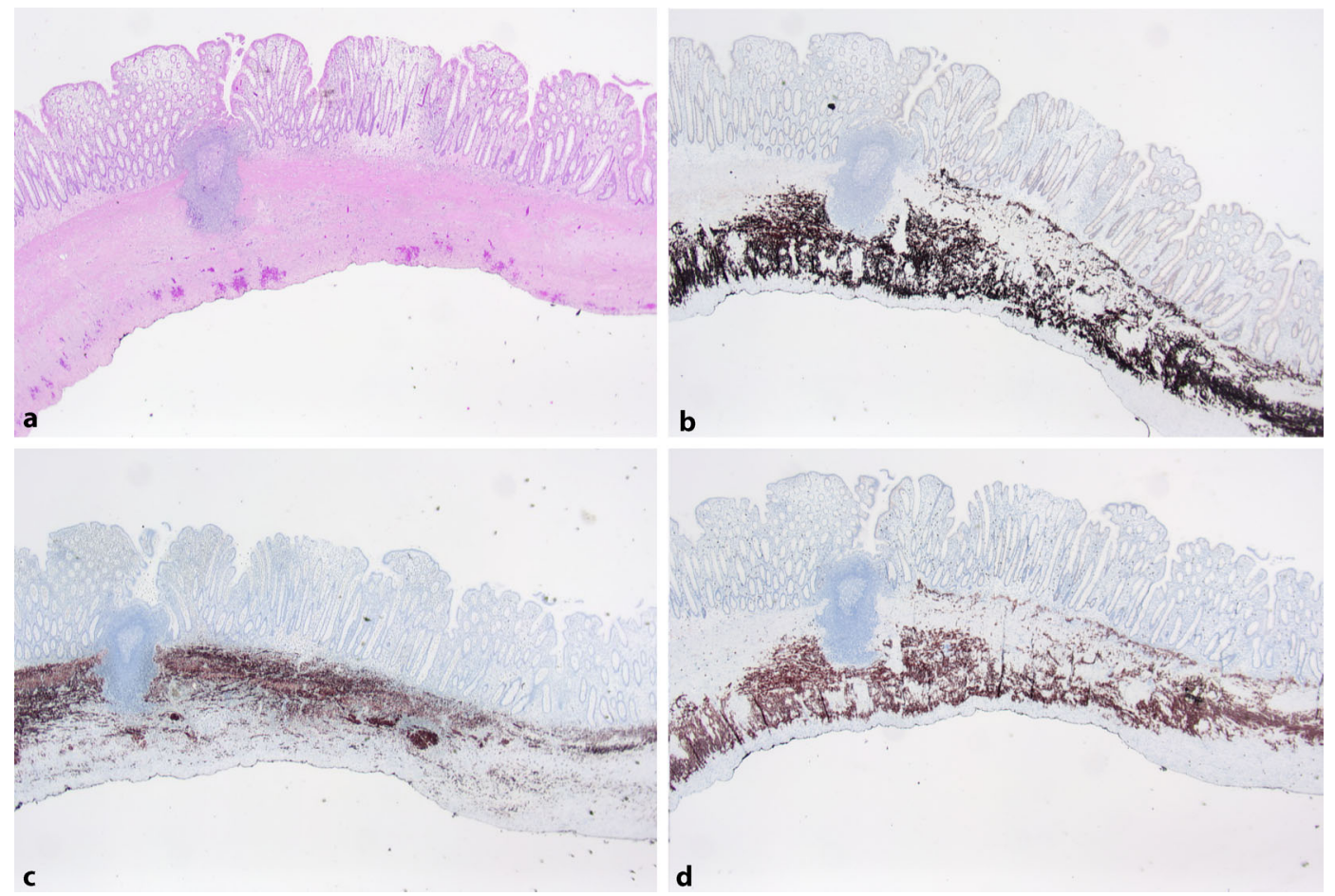

Abb. $2<$ Linearer gastrointestinaler Stromatumor (GIST) des lleums. Sehr selten können GIST auch eine lineares (statt ein noduläres) Wuchsmuster aufweisen (a, Übersichtsaufnahme, HE-Färbung, Vergrößerung 40:1). Die Tumorzellen exprimieren DOG-1 (b, Vergrößerung 40:1), CD34 (c, Vergrößerung 40:1) und CD117 (d, Vergrößerung 40:1)

liche onkogene Treiber Mutationen im KIT- oder PDGFRA-Gen nachzuweisen sind. BRAF-Mutationen (bislang stets p.V600E) sind demgegenüber viel seltener und machen vermutlich etwa $1-2 \%$ aus. Je sensitiver die Methoden zum Nachweis solcher Mutationen in den vergangenen Jahren wurden und je häufiger eine Anreicherung der Tumor-DNA z. B. durch Makro- oder Mikrodissektion erfolgte [11, 12], desto höher war auch die Frequenz der Mutationen bereits in kleinen GIST von $1 \mathrm{~cm}$ Größe und kleiner. Es handelt sich also um ein frühes pathogenetisches Ereignis, welches die autonome Proliferation von GIST-Vorläuferzellen antreibt. Interessant ist die Beobachtung, dass gerade bei kleinen, nicht nennenswert proliferationsaktiven und teilweise regressiv kalzifizierten GIST gehäuft seltenere Mutationstypen auftreten, deren onkogenes Potenzial offenbar geringer ist als das der GIST mit häufigeren Mutationstypen im KITExon 9 oder 11 [13]. Es ist gut vorstellbar, dass es sich also bei einer Subgruppe von GIST bedingt durch die Primärmutation in KIT oder PDGFRA um selbstlimitierende Neoplasien handelt. Unabhängig von der mutmaßlich tumorinitiieren- den Primärmutation erwerben GIST außerdem im Rahmen ihrer Progression weitere genomische Alterationen, z. B. in Form chromosomaler Gewinne und/ oder Verluste [14, 15], ohne die möglicherweise das Wachstum über eine gewisse Größe hinaus nicht möglich ist. Die meisten Studien zeigen in GIST zusätzlich zytogenetische Aberrationen, am häufigsten Verluste von 1p, 13q, $14 \mathrm{q}$, und $15 \mathrm{q}$ sowie „loss of heterozygosity“ (LOH) auf 22q, wobei dies v. a. GIST mit Primärmutationen in KIT oder PDGFRA, nicht aber die Subgruppe der GIST ohne diese Primärmutationen 
betrifft [16]. Bei Korrelation der Daten hochauflösender „comparative genomic hybridisation“ (CGH) mit Genexpressionsanalysen zeigt sich, dass bekannte Onkogene wie z. B. KRAS bei Chromosom-12p-Amplifikation hochreguliert sind, während Tumorsuppressorgene wie KIF1B, PPM1A und NF2 auf Chromosom $1 \mathrm{p}, 14 \mathrm{q}$ und $22 \mathrm{p}$ verloren gehen. In der sehr häufig von Alterationen betroffenen Region 14q23.1 konnten Tumorsuppressorgene wie DAAM1, RTN1 und DACT1 identifiziert werden [16]. Offenbar sind neben den bereits $\mathrm{zu}$ detektierenden KIT- oder PDGFRAMutationen also zusätzliche genomische oder epigenetische Aberrationen nötig, um die Progression mikroskopischer GIST voranzutreiben.

Somatische BRAF-Mutationen können als alternativer Pathomechanismus ebenfalls zur Entwicklung von GIST führen. Die Tumoren sind bevorzugt im Dünndarm lokalisiert und von unterschiedlichem biologischem Verhalten $[17,18]$. Die Wirksamkeit eines BRAF-Inhibitors bei einem metastasierten BRAF-mutierten GIST ist kasuistisch beschrieben [19].

\section{Syndromale GIST und GIST mit hereditärer Genese}

GIST mit familiärer Häufung oder in Assoziation mit weiteren Neoplasien können verschiedene genomische Hintergründe aufweisen: Keimbahnmutationen in KIT oder PDGFRA, die Neurofibromatose Typ 1 (NF1) oder Keimbahnmutationen im Succinatdehydrogenasekomplex.

Mittlerweile sind über 30 Familien beschrieben, die eine Keimbahnmutation im KIT-Gen aufweisen und neben GIST Mastozytosen, Hyperpigmentierungen und eine Dysphagie entwickeln können. Die KIT-Mutationen liegen in den typischen Hot-spot-Regionen des Gens (mit Ausnahme von KIT-Exon 9), das Auftreten zusätzliche Neoplasien hängt von der Lokalisation der KITMutation ab.

Die Frequenz von Keimbahnmutationen in PDGFRA ist deutlich geringer als in KIT mit nur wenigen beschriebenen Familien. Bemerkenswert ist in die-

Pathologe 2017-38:105-111 DOI 10.1007/s00292-017-0275-z

(c) Der/die Autor(en) 2017. Dieser Artikel ist eine Open-Access-Publikation.

E. Wardelmann - W. Hartmann · M. Trautmann · J. Sperveslage · S. Elges · E. Hekeler · S. Huss Gastrointestinale Stromatumoren des Magens und ihre Vorstufen

\section{Zusammenfassung}

Gastrointestinale Stromatumoren (GIST) sind die häufigsten Tumoren im Gastrointestinaltrakt, wenngleich sie hier deutlich seltener als epitheliale Tumoren vorkommen. In mehr als $60 \%$ der Fälle treten sie primär im Magen auf. Gerade kleine Läsionen mit einem Durchmesser von $\leq 1 \mathrm{~cm}$, sog. mikroskopische GIST, können multipel auftreten, nicht selten z. B. in der proximalen Magenwand und auch mitunter als Zufallsbefund bei Gastrektomien wegen eines synchronen Magenkarzinoms. Die Multizentrizität von GIST allein beweist weder ein metastatisches Verhalten, noch muss unbedingt von einer hereditären oder syndromalen Pathogenese ausgegangen werden. Mehrere sporadische synchrone oder metachrone GIST sind durch unterschiedliche Primärmutationen zumeist in KIT oder
PDGFRA gekennzeichnet und oft von wenig aggressiver Biologie. Möglicherweise ist ein Feldeffekt für das multiple Auftreten verantwortlich, auch bislang nicht identifizierte, die GIST-Entstehung begünstigende Noxen müssen erwogen werden. Bei fehlendem Nachweis von KIT- oder PDGFRA-Mutationen muss die Möglichkeit eines Succinatdehydrogenase(SDH)-defizienten GIST z. B. hereditär im Rahmen eines Carney-Stratakis-Syndroms oder syndromal im Rahmen einer CarneyTriade in Betracht gezogen werden.

Schlüsselwörter

Magen-GIST · KIT-Mutation · PDGFRaMutation · SDH-Mutation · Mikroskopischer GIST

\section{Gastrointestinal stromal tumors of the stomach and precursor lesions}

\section{Abstract}

Gastrointestinal stromal tumors (GIST) are the most common mesenchymal tumors in the gastrointestinal tract although they are much less frequent than epithelial tumors. In more than $60 \%$ of cases they occur in the stomach. Especially small lesions measuring $\leq 1 \mathrm{~cm}$ in diameter, so-called microscopic GIST can occur multifocally, frequently in the proximal stomach wall and sometimes as an incidental finding in a gastrectomy specimen resected for gastric cancer. The multicentricity of GIST alone is not proof of a metastatic behavior or a syndromal or hereditary disease. Multiple sporadic synchronous and metachronous GIST are characterized by different primary mutations mostly in the KIT or PDGFRA genes and are often less aggressive. It is speculative whether a field effect is responsible or whether still unknown GIST-promoting factors may facilitate the development of several independent lesions. If KIT or PDGFRA mutations are lacking, a succinate dehydrogenase (SDH) deficient GIST has to be considered, either hereditary as CarneyStratakis syndrome or syndromal as part of a Carney triad.

\section{Keywords}

Gastric GIST · KIT mutation P PDGFRA mutation · SDH mutation · Microscopic GIST sen Fällen das synchrone oder metachrone Auftreten inflammatorischer fibroider Polypen (IFP), die auch in der sporadischen Form häufig PDGFRA-Mutationen tragen $[20,21]$. Da außerdem gastrointestinale Lipome und große Hände bei einigen Patienten beobachtet werden können, wird mittlerweile auch vom „PDGFRA-mutant syndrome" gesprochen.

Bei Patienten mit NF1 ist das Risiko, an einem oder mehreren GIST zu erkranken, deutlich erhöht. Die Prävalenz von GIST bei NF1-Patienten liegt bei
$7 \%$, in Autopsiestudien sogar bei $25 \%$. Die Tumoren zeigen typischerweise keine Primärmutationen in KIT bzw. PDGFRA und treten oft multipel bevorzugt im Dünndarm auf. Die Mehrzahl der Tumoren verhält sich indolent, eine aggressivere Biologie wird in etwa 15-20\% der Fälle beobachtet $[22,23]$. Grundsätzlich ist es jedoch auch möglich, dass auch NF1Patienten „sporadische“ GIST mit den üblichen KIT- oder PDGFRA-Mutationen entwickeln. Solche Tumoren können dann ggf. auch wie sporadische GIST sys- 


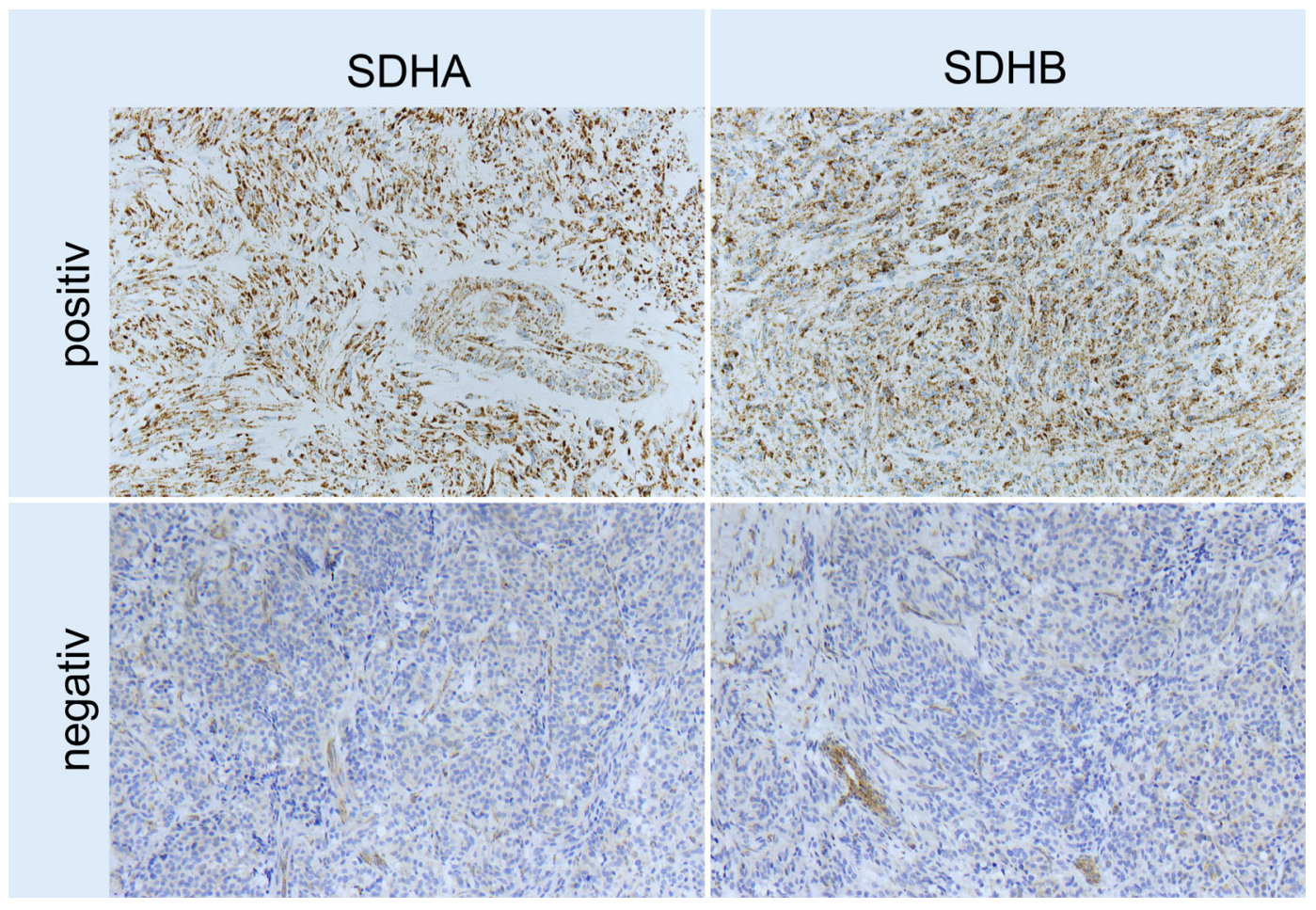

Abb. $3<$ Succinatdehydrogenase(SDH)-defiziente gastrointestinale Stromatumoren (GIST). SDHdefiziente GIST können immunhistochemisch unabhängig vom zugrunde liegenden Pathomechanismus am Ausfall von SDHB erkannt werden und müssen bei GIST ohne KIToder PDGFRA-Mutation erwogen werden (SDHA [Succinatdehydrogenase(SDH) Untereinheit A], Vergrößerung 100:1, SDHB [Succinatdehydrogenase(SDH) Untereinheit B], Vergrößerung 100:1) temisch therapiert werden. Somit kann auch hier eine KIT und PDGFRA Mutationsanalyse sinnvoll sein.

\section{Tumoren mit Succinat- dehydrogenasedefizienz}

Eine größere Gruppe unter den GIST ohne KIT- oder PDGFRA-Mutation nehmen die Tumoren mit Succinatdehydrogenase(SDH)-Defizienz ein. Diese kann durch eine inaktivierende Keimbahnmutation in einem der 4 Komplexpartner SDHA/B/C oder D bedingt sein, aber auch infolge einer epigenetischen Hypermethylierung von SDHC auftreten [24]. Bei Keimbahnmutation kommtes zusätzlich zu Paragangliomen (Carney-Stratakis-Syndrom), bei SDHC-Hypermethylierung außerdem zu pulmonalen Chondromen (Carney-Triade). Die CarneyTriade ist entsprechend ihres epigenetischen Hintergrundes nicht erblich und betrifft v. a. jüngere Frauen, die oft multinodulär wachsenden Tumoren sind im Magen lokalisiert und können lymphogen metastasieren. In allen beschriebenen Konstellationen dominieren epitheloide Tumoren im Magen, die immunhistochemisch einen SDHB-Verlust aufweisen unabhängig vom zugrunde liegenden
Pathomechanismus (• Abb. 3). Diese Eigenschaft teilen sie mit einer weiteren, nur inkomplett verstandenen GIST-Subgruppe, den pädiatrischen GIST. Diese treten bei Kindern und Jugendlichen auf, betreffen ebenfalls bevorzugt den Magen, metastasieren gehäuft lymphogen und zeigen eine epitheloide Morphologie.

Eine sehr gute aktuelle Übersicht zum Thema der hereditären Disposition und der syndromalen GIST findet sich bei Ricci [25].

\section{Bedeutung des synchronen Auftretens von mehr als einem GIST}

Bei mehr als einer Manifestion eines GIST wird im Allgemeinen von einem metastasierten Tumorleiden oder von einer hereditären Genese ausgegangen. Verschiedene Studien zeigen, dass diese Hypothese nicht zwangsläufig richtig ist, da in einem Teil der Fälle die verschiedenen Tumormanifestationen unterschiedliche Primärmutationen in KIT bzw. PDGFRA aufweisen und sich somit als sporadisch erweisen. Oft sind dies eher kleine, wenig aggressive GIST, die nicht unbedingt im selben
Abschnitt des Gastrointestinaltrakts auftreten. Bevorzugt betroffen sind Magen und Dünndarm. Insbesondere in der proximalen Magenwand werden zudem mitunter auch mehrere mikroskopische GIST als Zufallsbefund in Gastrektomien bei simultan bestehendem Magenkarzinom beobachtet. Inwieweit hier pathogenetische Zusammenhänge zwischen der mesenchymalen und epithelialen Tumorgenese bestehen, ist ungeklärt. Da die in diesen Fällen auftretenden GIST zumeist klein sind und nur ein geringes Rückfallrisiko besitzen, verschlechtert sich die Prognose der zumeist älteren Patienten mit synchronem Magenkarzinom durch die GIST nicht $[26,27]$.

In einer eigenen Untersuchung von knapp 1000 konsekutiven GIST konnten multiple Tumoren (definiert als 2 GIST oder mehr) ohne Anhaltspunkte für eine hereditäre Genese in 17 Fällen nachgewiesen werden, davon in 16 Fällen synchron, in einem Fall metachron (bislang nicht publizierte Daten). Die Primärmutationen waren in diesen GIST jeweils unterschiedlich und betrafen sowohl das KIT- als auch das PDGFRA-Gen. Wie es zu dem ungewöhnlichen Auftreten gleich mehrerer derart seltener Tumoren vermutlich unabhängig voneinander kom- 

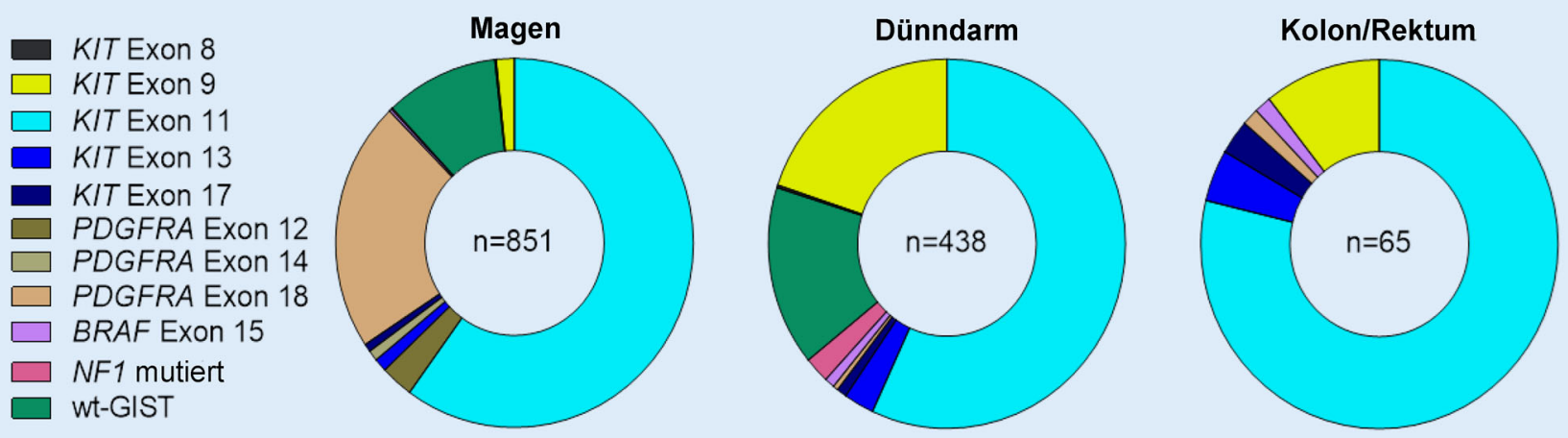

Abb. 4 ॥ Mutationsdaten aus dem Weichgewebstumorregister NRW mit Daten von 1364 GIST getrennt nach Lokalisation und Mutation. Es ist exemplarisch gut erkennbar, dass PDGFRA-Mutationen (Brauntöne) im Magen viel häufiger als im Dünnoder Dickdarm vorkommen. wt-GIST gastrointestinaler Stromatumor, Wildtyp

men kann, ist pathogenetisch ungeklärt. Diskutiert wird ein Feldeffekt durch eine oder mehrere bislang nicht identifizierte Noxen ebenso wie eine mögliche Prädisposition der bestimmten Klone der ICC. Auch genomische Mosaike z. B. mit fokalen Alterationen im NF1-Gen im Gastrointestinaltrakt wurden bereits erwogen, ohne dass bislang eine dieser Theorien substanziell unterfüttert werden konnte [28-32]. In jedem Fall sollte die getrennte genomische Charakterisierung mehrerer Tumormanifestationen erwogen werden, wenn es sich nur um wenige Läsionen handelt oder ein metastasiertes Tumorleiden eher unwahrscheinlich ist.

\section{Prognose von Magen-GIST}

GIST des Magens zeigen insgesamt einen günstigeren klinischen Verlauf als solche anderer Primärlokalisation. In einer retrospektiven Studie an fast 2000 GIST konnten Miettinen und Lasota [9] vom Armed Forces Institute of Pathology (AFIP) dies eindrucksvoll zeigen. Danach weisen auch große, wenig proliferationsaktive Magen-GIST einen günstigeren klinischen Verlauf auf als gleich große Tumoren anderer Primärlokalisation, umgekehrt sind proliferationsaktive, außerhalb des Magens lokalisierte Tumoren auch bei geringerer Größe häufig schon aggressiv. Die Ermittlung der Proliferation anhand der Mitosenzahl erfolgt heute nicht mehr an 50 HPF (,high power field"), sondern in einer Fläche von $5 \mathrm{~mm}^{2}$, was bei modernen Mikroskopen zumeist weniger als $20 \mathrm{HPF}$ entspricht. Ein Grund für den günstigeren Verlauf der Magen-GIST könnte das im Vergleich $\mathrm{zu}$ anderen Lokalisationen anzutreffende Mutationsspektrum sein, da im Magen über $20 \%$ der Fälle eine prognostisch günstigere PDGFRA-Mutation aufweisen, die in anderen Lokalisationen nur äußerst selten vorkommt. Entsprechende Mutationsdaten von 1365 primären GIST aus unserem Register sind in $\bullet$ Abb. 4 getrennt nach den Lokalisationen Magen, Dünndarm und Kolorektum gezeigt [33, 34].

Auch wegen der prognostischen Relevanz sollte die Mutationsanalyse in GIST zumindest in den Schwerpunktregionen von KIT und PDGFRA heute zum Standard gehören, da auch die Therapieplanung ohne diese nur schwerlich möglich ist. Gerade in Magen-GIST ist in mehr als $20 \%$ der Fälle die zur Imatinibresistenz führende Punktmutation im PDGFRAExon 18 p.D842V zu erwarten. Bei GIST außerhalb des Magens wird bei nachgewiesener KIT-Exon-9-Mutation die Imatinibdosis sowohl adjuvant als auch bei metastasiertem Tumorleiden auf $800 \mathrm{mg}$ verdoppelt.

Hier steht eine Anzeige.

Springer 


\section{Wichtige Differenzialdiagnosen zu Magen-GIST}

Differenzialdiagnostisch müssen bei einer spindelzelligen oder epitheloiden Neoplasie der Magenwand verschiedene Tumoren ausgeschlossen werden, was mit Einsatz eines entsprechenden immunhistochemischen Panels oft gelingt. Leiomyome, die lediglich im distalen Ösophagus und im proximalen $\mathrm{Ma}$ gen häufiger sind als GIST, exprimieren durchgängig und kräftig Desmin. Eingeschlossene, nicht selten zahlreiche CD117-positive Mastzellen sind gelegentlich irreführend, können aber im Zweifel durch Nachweis von Mastzelltryptase identifiziert werden. Schwannome sind kräftig positiv für S100Protein und häufig von einem dichten lymphozytären Randwall umgeben. Inflammatorische fibroide Polypen sind zwar wie viele Magen-GIST CD34-positiv, aber negativ für CD117 und DOG1, den sensitivsten GIST-Marker. Da diese Tumoren jedoch gleichartige PDGFRAMutationen wie GIST aufweisen können, ist zu bedenken, dass ein vermeintlich „klassischer Mutationsnachweis“ in PDGFRA eine GIST-Fehldiagnose resultieren kann [35]. Inflammatorische myofibroblastische Tumoren exprimieren häufig ALK und zeigen zudem ALKoder ROS1-Translokationen. Auch sie sind negativ für CD117 und DOG1. Dies gilt auch für abdominelle Fibromatosen, die nukleär $\beta$-Catenin exprimieren und häufig CTNNB1-Mutationen aufweisen [36]. Dedifferenzierte Liposarkome exprimieren MDM2 und CDK4 und zeigen entsprechende Clusteramplifikationen. Der Einsatz mehrerer immunhistochemischer Marker ist also im Zweifel empfehlenswert. Das Minimalpanel für GIST sollte CD117, DOG1 und Ki67 umfassen, zudem ggf. SDHB.

\section{Fazit für die Praxis}

- Auch sehr kleine, sog. mikroskopische GIST $\leq 1 \mathrm{~cm}$ tragen oft aktivierende Mutationen in KIT oder PDGFRA und haben daher als echte Neoplasien zu gelten.

- Die Multizentrizität allein ist kein ausreichender Beleg für eine hereditäre oder syndromale Genese oder ein metastasiertes GIST-Leiden.

- Die Mutationsanalyse - bei GIST mit signifikantem Rückfallrisiko mittlerweile Standard - kann im Zweifel bei multiplen synchronen GIST-Manifestationen bei der Klärung helfen.

- Ein SDH-defizienter GIST kann immunhistochemisch am Ausfall von SDHB erkannt werden und muss bei GIST ohne KIT- oder PDGFRAMutation erwogen werden.

- Magen-GIST haben eine günstigere Prognose als vergleichbare Tumoren anderer Primärlokalisation, möglicherweise durch einen höheren Anteil PDGFRA-mutierter Läsionen.

\section{Korrespondenzadresse}

\section{Prof. Dr. E. Wardelmann}

Gerhard-Domagk-Institut für Pathologie, Universitätsklinikum Münster

Albert-Schweitzer-Campus 1, Gebäude D17, 48149 Münster, Deutschland eva.wardelmann@ukmuenster.de

\section{Einhaltung ethischer Richtlinien}

Interessenkonflikt. E. Wardelmann, W. Hartmann, M. Trautmann, J. Sperveslage, S. Elges, E. Hekeler und $S$. Huss geben an, dass kein Interessenkonflikt besteht.

Dieser Beitrag beinhaltet keine von den Autoren durchgeführten Studien an Menschen oder Tieren.

Open Access. Dieser Artikel wird unter der Creative Commons Namensnennung 4.0 International Lizenz (http://creativecommons.org/licenses/by/4.0/deed. de) veröffentlicht, welche die Nutzung, Vervielfältigung, Bearbeitung, Verbreitung und Wiedergabe in jeglichem Medium und Format erlaubt, sofern Sie den/die ursprünglichen Autor(en) und die Quelle ordnungsgemäß nennen, einen Linkzur Creative Commons Lizenz beifügen und angeben, ob Änderungen vorgenommen wurden.

\section{Literatur}

1. Agaimy A, Wünsch PH (2006) Gastrointestinal stromal tumours: a regular origin in the muscularis propria, but an extremely diverse gross presentation. A review of 200 cases to critically re-evaluate the concept of so-called extra-gastrointestinal stromal tumours. Langenbecks Arch Surg 391:322-329

2. Kawanowa K, Sakuma Y, Sakurai S et al (2006) High incidence of microscopic gastrointestinal stromal tumors in the stomach. Hum Pathol 37:1527-1535

3. Agaimy $A$, Wünsch $P H$, Hofstaedter $F$ et al (2007) Minute gastrics clerosing stromal tumors (GIST tumorlets) are common in adults and frequently show c-KIT mutations. Am J Surg Pathol 31:113-120

4. Nilsson B, Bümming $P$, Meis-Kindblom JM et al (2005) Gastrointestinal stromal tumors: the incidence, prevalence, clinical course, and prognostication in the preimatinib mesylate era a population-based study in western Sweden. Cancer 103:821-829

5. Goettsch WG, Bos SD, Breekveldt-Postma N et al (2005) Incidence of gastrointestinal stromal tumours is underestimated: results of a nationwide study. Eur J Cancer 41:2868-2872

6. Chen H, Hirota S, Isozaki K et al (2002) Polyclonal nature of diffuse proliferation of interstitial cells of Cajal in patients with familial and multiple gastrointestinal stromal tumors. Gut 51:793-796

7. Agaimy A, Wünsch PH (2006) Sporadic Cajal cell hyperplasia is common in resection specimens for distal oesophageal carcinoma. A retrospective review of 77 consecutive surgical resection specimens. Virchows Arch 448:288-294

8. Agaimy A, Wünsch PH, Dirnhofer S et al (2008) (2008) Microscopic gastrointestinal stromal tumors in esophageal and intestinal surgical resection specimens: a clinicopathologic, immunohistochemical, and molecular study of 19 lesions. Am J Surg Pathol 32(6):867-873

9. Miettinen M, Lasota J (2006) Gastrointestinal stromal tumors: pathology and prognosis at different sites. Semin Diagn Pathol 23:70-83

10. Agaimy A, Märkl B, Arnholdt H, Hartmann A, Schneider-Stock R, Chetty R (2010) Sporadic segmental Interstitial cell of cajal hyperplasia (microscopic GIST) with unusual diffuse longitudinal growth replacing the muscularis propria: differential diagnosis to hereditary GIST syndromes. Int J Clin Exp Pathol 3(5):549-556

11. Merkelbach-Bruse $S$, Dietmaier W, Füzesi $L$ et al (2010) Pitfalls in mutational testing and reporting of common KIT and PDGFRA mutations in gastrointestinal stromal tumors. BMC Med Genet 11:106

12. Corless CL, McGreevey L, Haley A et al (2002) KIT mutations are common in incidental gastrointestinal stromal tumors one centimeter or less in size. Am J Pathol 160:1567-1572

13. Rossi S, Gasparotto D, Toffolatti L et al (2010) Molecular and clinicopathologic characterization of gastrointestinal stromal tumors (GISTs) of small size. Am J Surg Pathol 34(10):1480-1491

14. Assämäki R, Sarlomo-Rikala M, Lopez-Guerrero JA et al (2007) Array comparative genomic hybridization analysis of chromosomal imbalances and their target genes in gastrointestinal stromal tumors. Genes Chromosomes Cancer 46:564-576

15. Wozniak A, Sciot R, Guillou L et al (2007) Array CGH analysis in primary gastrointestinal stromal tumors: cytogenetic profile correlates with anatomic site and tumor aggressiveness, irrespective of mutational status. Genes Chromosomes Cancer 46:261-276. doi:10.1002/gcc.20408

16. Astolfi A, Nannini M, Pantaleo MA et al (2010) A molecular portrait of gastrointestinal stromal tumors: an integrative analysis of gene expression profiling and high-resolution genomic copy number. Lab Invest 90:1285-1294

17. Agaram NP, Wong GC, Guo T et al (2008) Novel V600E BRAF mutations in imatinib-naive and imatinib-resistant gastrointestinal stromal tumors. Genes Chromosomes Cancer 47:853-859

18. Huss S, Pasternack H, Ihle MA, Merkelbach-Bruse $S$, Heitkötter B, Hartmann W, Trautmann M, Gevensleben $\mathrm{H}$, Büttner R, Schildhaus HU, Wardelmann E (2017) Clinicopathological and molecular features 
of a large cohort of gastrointestinal stromal tumors (GISTs) and review of the literature:BRAF mutations in KIT/PDGFRA wildtype GISTs are rare events. Hum Pathol. doi:10.1016/j.humpath.2017.01.005 [Epub ahead of print]

19. Falchook GS, Trent JC, Heinrich MC et al (2013) BRAF Mutant Gastrointestinal Stromal Tumor: First report of regression with BRAF inhibitor dabrafenib (GSK2118436) and whole exomic sequencing for analysis of acquired resistance. Oncotarget 4:310-315

20. Schildhaus HU, Cavlar T, Binot E et al (2008) Inflammatory fibroid polyps harbour mutations in the platelet-derived growth factor receptor alpha (PDGFRA) gene.J Pathol 216(2):176-182

21. Huss S, Wardelmann E, Goltz D et al (2012) Activating PDGFRA mutations in inflammatory fibroid polyps occur in exons 12,14 and 18 and are associated with tumour localization. Histopathology 61:59-68

22. Miettinen M, Fetsch JF, Sobin LH, Lasota J (2006) Gastrointestinal stromal tumors in patients with neurofibromatosis 1: a clinicopathologic and molecular genetic study of 45 cases. Am J Surg Pathol 30:90-96

23. Mussi C, Schildhaus HU, Gronchi A et al (2008) Therapeuticconsequencesfrommolecularbiology for gastrointestinal stromal tumor patients affected by neurofibromatosis type 1. Clin Cancer Res 14:4550-4555

24. Haller F, Moskalev EA, Faucz FR et al (2014) Aberrant DNA hypermethylation of SDHC: a novel mechanism of tumor development in Carney triad. Endocr Relat Cancer 21(4):567-577

25. Ricci R (2016) Syndromic gastrointestinal stromal tumors. Hered Cancer Clin Pract 14:15

26. Du J, Shen N, He HS, Fu XLet al (2016) Synchronous gastrointestinal cancer and gastrointestinal stromal tumors: a single-institution experience. World J Surg Oncol 14:130

27. LiuZ, Liu S, Zheng Getal (2016) Clinicopathological features and prognosis of coexistence of gastric gastrointestinal stromal tumor and gastric cancer. Medicine (Baltimore) 95:45

28. Haller F, Schulten HJ, Armbrust T et al (2007) Multicentric sporadic gastrointestinal stromal tumors (GISTs) of the stomach with distinct clonal origin: differential diagnosis to familial and syndromal GIST variants and peritoneal metastasis. Am JSurg Pathol 31:933-937

29. Kang DY, Park CK, Choi JS et al (2007) Multiple gastrointestinal stromal tumors: clinicopathologic and genetic analysis of 12 patients. Am J Surg Pathol 31:224-232

30. Gasparotto D, Rossi S, Bearzi l et al (2008) Multiple primary sporadic gastrointestinal stromal tumors in the adult: an underestimated entity. Clin Cancer Res 14(18):5715-5721

31. Agaimy A, Dirnhofer S, Wünsch PH et al (2008) Multiple sporadic gastrointestinal stromal tumors (GISTs) of the proximal stomach are caused by different somatic KIT mutations suggesting a field effect. Am J Surg Pathol 32(10):1553-1559

32. Chen X, Ya-Lan L, Hong-Yu Y et al (2012) Synchronous and metachronous multiple gastrointestinal stromal tumors. Histol Histopathol 27:225-234

33. Huss S, Kunstlinger $\mathrm{H}$, Wardelmann E et al (2013) A subset of gastrointestinal stromal tumors previously regarded as wild-type tumors carries somatic activating mutations in KIT exon 8 (p.D419del). Mod Pathol 26:1004-1012

34. Kunstlinger $\mathrm{H}$, Huss $\mathrm{S}$, Merkelbach-Bruse $\mathrm{S}$ et al (2013) Gastrointestinal stromal tumors with KIT exon 9 mutations:updateongenotype-phenotype correlation and validation of a high-resolution melting assay for mutational testing. Am J Surg Pathol 37:1648-1659

35. Huss $S$, Wardelmann E, Goltz D, Binot E, Hartmann W, Merkelbach-Bruse S, Buttner R, Schildhaus HU (2012) Activating PDGFRA mutations in inflammatory fibroid polyps occur in exons 12,14 and 18 and are associated with tumour localization. Histopathology 61:59-68

36. Huss S, Nehles J, Binot E et al (2013) Beta-catenin (CTNNB1) mutations and clinicopathological features of mesenteric desmoid-type fibromatosis. Histopathology 62:294-304

\section{Diagnosen: Wann sind mehrere Ärzte besser?}

Kollektive Intelligenz ist ein vielversprechender Ansatz, um bessere ärztliche Entscheidungen zu treffen. Das konnten Studien des Max-Planck-Instituts für Bildungsforschung und des Leibniz-Instituts für Gewässerökologie und Binnenfischerei bereits zeigen. In einer Folgestudie untersuchten die Wissenschaftler nun, welche Voraussetzungen erfüllt sein müssen, damit die Gruppe erfolgreicher ist als der beste Einzelne in der Gruppe. Dabei zeigte sich, dass sich die Ärzte hinsichtlich ihrer Diagnosegenauigkeit ähneln müssen. Nur so können die kombinierten Entscheidungen mehrerer Ärzte die Entscheidung des besten Arztes der Gruppe überflügeln. Dieser Effekt zeigt sich auch bei verschiedenen Gruppengrößen oder unterschiedlichen Leistungsniveaus des besten Arztes innerhalb der Gruppe.

Für ihre Studie berechneten die Wissenschaftler anhand von über 20.000 Bewertungen von mehr als 140 Ärzten die Diagnosegenauigkeit der einzelnen Ärzte. Mit diesen Informationen simulierten sie, unter welchen Bedingungen die mittels Regeln der kollektiven Intelligenz kombinierten Diagnosen treffsicherer sind als Einzeldiagnosen. Das Ergebnis unterstreiche die Bedeutung der Diagnosegenauigkeit der einzelnen Entscheider für das Gesamtergebnis. Dies sollte auch in der Praxis berücksichtigt werden - beispielsweise bei der unabhängigen Doppelbefundung einer Mammografie-Aufnahme durch zwei Ärzte, so die Studienautoren.

Literatur: Kurvers RHJM, Herzog SM, Hertwig R et al (2016) Boosting medical diagnostics by pooling independent judgments. PNAS 8777-8782

Quelle: Max-Planck-Institut für Bildungsforschung www.mpib-berlin.mpg.de 